\title{
Combining Ability Studies in CMS based and Conventional Hybrids of Cotton (G. hirsutum)
}

\author{
G.R. Gopal ${ }^{1}$, D.B. Deosarkar ${ }^{2}$ and V.N. Chinchane ${ }^{3 *}$ \\ Department of Agricultural Botany (Genetics and Plant Breeding), \\ Vasantrao Naike Marathawada Krishi Vidyapeeth, Parbhani. 431402 (M.S.), India \\ *Corresponding author
}

\begin{tabular}{|l|}
\hline Ke y w o r d s \\
Combining ability, \\
GCA, SCA, Yield, \\
Fibre strength
\end{tabular}

\section{Introduction}

Cotton is being cultivated in 70 countries of the world with a total coverage of $33.14 \mathrm{~m}$ ha. China, India, USA and Pakistan are the major cotton producing countries in the world accounting for 70 per cent of the world's cotton area and production. India is the largest cotton growing country in the world with
35.29 per cent of world cotton area followed by China $(15.23 \%)$. China and India are the major cotton consuming countries in the world (around 55\%). USA and India constitute 27 and 19.5 per cent of the world's cotton exports respectively. There are four cultivated species of cotton viz. Gossypium arboreum, G.herbaceum, G.hirsutumand G.barbadense. The first two species are diploid $(2 n=26)$ and 
are native to old world. They are also known as Asiatic cottons because they are grown in Asia. The last two species are tetraploid $(2 \mathrm{n}=52)$ and are also referred to as New World Cottons. G.hirsutumis also known as American cotton or upland cotton and G.barbadenseas Egyptian cotton or Sea Island cotton or Peruvian Cotton or Tanguish Cotton or quality cotton. G.hirsutumis the predominant species which alone contributes about $90 \%$ to the global production. Perhaps, India is the only country in the world where all the four cultivated species are grown on commercial scale.

\section{Materials and Methods}

The present study comprised of three male sterile CMS lines, three maintainer lines and ten male fertile males (testers/ restorers) with three standard checks in two sets i.e. CMS and conventional thus making $60 \mathrm{~F}_{1} \mathrm{~s}(30 \mathrm{CMS}$ and 30 conventional) using Line $\mathrm{x}$ Tester mating design. These lines, testers and hybrids along with three checks were sown during kharif, 2016 at three locations viz., Cotton Research Station, Nanded (L-1), Experimental farm of Department of Agricultural Botany, VNMKV, Parbhani (L-2) and Experimental farm, Agricultural Research Station, Badnapur (L3 ). The observations recorded on days to $50 \%$ flowering, plant height $(\mathrm{cm})$, number of sympodia per plant, days to $50 \%$ boll burst, earliness index, number of sympodia per plant, number of boll per plant, boll weight $(\mathrm{g})$, seed cotton yield per plant $(\mathrm{g})$, seed index $(\mathrm{g})$, lint index $(\mathrm{g})$, harvest index $(\%)$, seed cotton yield per hectare $(\mathrm{kg})$, ginning percentage, upper half mean length $(\mathrm{mm})$, uniformity index $(\%)$, micronaire value (ug/inch) and fibre strength (g/tex).

\section{Results and Discussion}

The pooled analysis of variance for line $\mathrm{x}$ tester was found significant for all the characters. The significance of location $\mathrm{x}$ crosses interaction for plant height, days to 50 $\%$ boll burst, number of sympodia per plant, number of bolls per plant, boll weight, seed cotton yield per plant, harvest index, seed cotton yield per hectare and ginning percentage showed the diversity between the environments.

The location $x$ line effects were found significant for plant height, days to $50 \%$ boll burst, number of bolls per plant, uniformity index and micronaire value. The significance of location $\mathrm{x}$ line $\mathrm{x}$ tester interaction effect for plant height, number of monopodia per plant, days to $50 \%$ boll burst, number of sympodia per plant, number of boll per plant, boll weight, seed cotton yield per plant, lint index, harvest index, seed cotton yield per hectare, ginning percentage indicated higher interaction of non-additive genetic effects with environment. This could be the reason for difference in heterosis over the environment for yield (Table 1).

\section{GCA effect of parents}

The lines CAK 53A, CAK 53B and tester DHY-286-1R had the highest GCA effect for the traits days to $50 \%$ flowering and days to $50 \%$ boll burst. The tester AKH-07R possessed the highest GCA effect for the seed cotton yield per hectare and also exhibited high GCA (in desirable direction) for the traits, number of sympodia, number of bolls per, harvest index and cotton seed yield per hectare. The line CAK 23 B reported the high GCA effect to the traits, earliness index, number of bolls per plant, boll weight, seed cotton yield per plant, seed cotton yield per hectare, ginning percentage and fiber strength. The tester, R-2000-23 showed highest GCA effect for characters fiber strength, uniformity index and ginning percentage also possessed the significant (in desirable direction) high GCA effect to the traits i.e. upper half mean 
length, seed cotton yield per plant and number of bolls per plant. Hence these genotypes can be used in future for improvement of fiber properties.

The line CAK 53 B exhibited the highest GCA effect for the character upper half mean length, uniformity index and number of bolls per plant also showed the second position among line for the traits fiber strength, days to $50 \%$ flowering and days to $50 \%$ boll burst. For micronaire value the high GCA effect is showed by line CAK 53 A and tester R-104413.

\section{SCA effect of crosses}

In the CMS set of crosses, according to the pooled data the highest SCA effects were observed for the cross combination CAK 23A $x$ R-42-8 (42.89) which also exhibited good performance in seed yield. It also exhibited the highest SCA effect for the traits, days to $50 \%$ flowering, days to $50 \%$ boll burst, earliness index, seed index, cotton seed yield per hectare. The CMS cross, CAK 53 A x AKH$07 \mathrm{R}$ possessed the highest SCA for the traits like number of sympodia per plant (3.32), number of bolls per plant (0.74), boll weight(0.74), seed cotton yield per plant (33.72) and it showed highest per se performance for the seed cotton yield per plant, ginning percentage, lint index and harvest index. In the conventional set of crosses, the highest SCA effect for seed cotton yield per plant observed for crosses CAK 23B $x$ DHY-286-1R (47.99) with high mean performance. It had also recorded high SCA effect in the earliness index and number of bolls per plant. The cross combination CAK 23B X R-2000-23 exhibited the highest SCA effect for number of bolls per plant, number of sympodia per plant and plant height.

For the fiber traits in CMS, highest SCA effects were recorded by SRT-1A x R-2000-
23 for fiber strength (3.59) upper half mean length (1.05). In the conventional hybrids, CAK 53 B x R-2000-23 (3.39) exhibited the highest SCA effect for the fiber strength and high per se performance to the seed cotton yield.

\section{Nature of gene action}

The ratio of GCA and SCA i.e. predictability ratio of micronaire value indicating the predominantly additive gene action in expression of the characters. This ratio for number of sympodia per plant and boll weight was greater than 0.50 but less than 0.80 indicated that the equal importance of the additive as well as non-additive gene action, while rest of the traits possessed the lower predictability ratio than 0.50 indicating the non-additive gene action as the prime cause in the expression of these traits. So while applying the selection in the further generations, gene action controlling the characters should be taken into consideration viz. where fixable component i.e. additive gene action is evident, the selection in the further generations would be rewarding after judging the positive correlation of the characters under investigation with the yield. In the characters where non fixable component is predominant heterosis breeding and recombination breeding with postponement of selection at later generation would be feasible (Laxman and Pradeep, 2003.)

\section{Selection of promising parent and crosses}

The lines CAK 23B and SRT-1B appeared to be the best general combiner for seed cotton yield and some fibre properties which may do well in cross combination with other parents. The tester AKH-07 R found to be best general combiner for the seed cotton yield. The tester R-2000-23 was found best general combiner for most of the fibre properties with high seed cotton yield. 
Table.1 Pooled estimates of GCA effects of parents and SCA effects of crosses studied over three environments

\begin{tabular}{|c|c|c|c|c|c|c|c|c|c|c|}
\hline $\begin{array}{l}\text { Sr. } \\
\text { No. }\end{array}$ & Genotype & $\begin{array}{c}\text { Days to } 50 \\
\% \\
\text { flowering }\end{array}$ & $\begin{array}{l}\text { Plant } \\
\text { height } \\
(\mathrm{cm})\end{array}$ & $\begin{array}{c}\text { No. of } \\
\text { monopodia } \\
\text { per plant }\end{array}$ & $\begin{array}{l}\text { Days to } \\
50 \% \\
\text { boll burst }\end{array}$ & $\begin{array}{l}\text { Earliness } \\
\text { index }\end{array}$ & $\begin{array}{c}\text { No. of } \\
\text { sympodia } \\
\text { per plant }\end{array}$ & $\begin{array}{c}\text { No. of bolls } \\
\text { per plant }\end{array}$ & $\begin{array}{c}\text { Boll } \\
\text { weight } \\
\text { (g) }\end{array}$ & $\begin{array}{c}\text { Seed cotton } \\
\text { yield } \\
\text { per plant }(\mathrm{g})\end{array}$ \\
\hline & GCA Lines & & & & & & & & & \\
\hline 1 & CAK 53A & $-3.78 * *$ & -1.12 & 0.04 & $-1.8 * *$ & $-0.009 *$ & $0.82 * *$ & $-1.39 *$ & $-0.21 * *$ & $-7.99 * *$ \\
\hline 2 & CAK 53B & $-3.05 * *$ & $-2.19 *$ & $0.12 *$ & $-0.98 * *$ & $-0.008 *$ & 0.21 & $2.95 * *$ & -0.05 & -0.24 \\
\hline 3 & CAK 23A & $0.71 * *$ & $-2.77 * *$ & -0.084 & $0.41 *$ & -0.001 & 0.11 & $-2.92 * *$ & 0.01 & $-8.68 * *$ \\
\hline 4 & CAK 23B & $0.96 * *$ & $2.49 * *$ & 0.06 & -0.18 & $0.016 * *$ & $1.01 * *$ & $2.90 * *$ & $0.32 * *$ & $12.49 * *$ \\
\hline 5 & SRT-1A & $3.56 * *$ & -0.938 & -0.04 & $1.43 * *$ & 0.004 & $-1.37 * *$ & $-2.39 * *$ & -0.08 & $-4.59 * *$ \\
\hline \multirow[t]{2}{*}{6} & SRT-1B & $1.58 * *$ & $4.54 * *$ & -0.10 & $1.11 * *$ & -0.002 & $-0.79 * *$ & 0.85 & 0.02 & $9.02 * *$ \\
\hline & GCA Testers & & & & & & & & & \\
\hline 1 & $\mathrm{R}-42-8$ & 0.21 & $-2.70 *$ & 0.05 & 0.06 & -0.006 & $-1.49 * *$ & -1.09 & $-0.19 *$ & -2.00 \\
\hline 2 & R-2000-23 & $0.48 *$ & 0.46 & 0.01 & -0.40 & -0.004 & 0.70 & $2.35 * *$ & $0.28 * *$ & $14.56^{* *}$ \\
\hline 3 & R- 2000-17-2 & $-0.73 * *$ & 0.27 & -0.07 & 0.40 & 0.010 & -0.69 & 0.57 & $0.18 *$ & $-9.29 * *$ \\
\hline 4 & R-2000-26 & -0.28 & $3.21 * *$ & -0.07 & $0.81 * *$ & $-0.032 * *$ & -0.06 & $1.90 *$ & 0.10 & $4.00 * *$ \\
\hline 5 & R-1044-13 & $1.26 * *$ & $-3.67 * *$ & 0.09 & -0.07 & $0.018 * *$ & 0.02 & 0.72 & -0.01 & 1.77 \\
\hline 6 & R-2000-21 & -0.20 & -1.07 & 0.004 & $-1.01 * *$ & -0.006 & 0.30 & $-3.38 * *$ & 0.05 & $-10.81 * *$ \\
\hline 7 & $\mathrm{R}-23$ & $1.23 * *$ & $4.25 * *$ & -0.05 & $0.78 * *$ & $0.029 * *$ & -0.27 & $-3.93 * *$ & -0.14 & $-14.53 * *$ \\
\hline 8 & $\mathrm{R}-53$ & -0.03 & -0.95 & -0.06 & 0.09 & $-0.021 * *$ & -0.30 & -0.93 & -0.11 & 0.93 \\
\hline 9 & AKH-07R & $1.04 * *$ & 1.07 & -0.06 & 0.40 & $0.019 * *$ & $1.59 * *$ & $5.19 * *$ & 0.12 & $29.56^{* *}$ \\
\hline \multirow[t]{2}{*}{10} & DHY-286-1R & $-2.98 * *$ & -0.86 & $0.16^{*}$ & $-1.07 * *$ & -0.007 & 0.20 & -1.40 & $-0.29 * *$ & $-14.18 * *$ \\
\hline & SCA Crosses & & & & & & & & & \\
\hline 1 & CAK 53A X R-42-8 & $7.08 * *$ & 3.56 & -0.18 & $3.46 * *$ & 0.015 & 1.82 & 0.84 & -0.17 & $-16.25 * *$ \\
\hline 2 & CAK 53A X R-2000-23 & $1.64 * *$ & -5.43 & -0.13 & -1.06 & 0.022 & $-2.21 *$ & -3.44 & -0.14 & $-28.49 * *$ \\
\hline 3 & CAK 53A X R- 2000-17-2 & $-3.96 * *$ & 1.86 & $-0.42 *$ & $-1.86 * *$ & -0.002 & -0.81 & -0.28 & -0.02 & $-6.80 *$ \\
\hline 4 & CAK 53A X R-2000-26 & $-6.41 * *$ & -2.84 & -0.28 & $-2.78 * *$ & 0.022 & -0.02 & -2.79 & -0.08 & $22.36 * *$ \\
\hline 5 & CAK 53A X R-1044-13 & $1.53 * *$ & 2.45 & -0.21 & 0.60 & $-0.032 *$ & -0.09 & -1.67 & -0.37 & $14.04 * *$ \\
\hline 6 & CAK 53A X R-2000-21 & $2.67 * *$ & -0.08 & 0.11 & $1.38 *$ & 0.021 & -0.38 & 1.49 & 0.082 & -4.67 \\
\hline 7 & CAK 53A X R-23 & $1.89 * *$ & 3.46 & $0.60 * *$ & -0.25 & $-0.027 *$ & -0.79 & -2.41 & -0.133 & 4.92 \\
\hline 8 & CAK 53A X R-53 & $-2.99 * *$ & 2.81 & 0.14 & 0.43 & -0.003 & -1.09 & -2.05 & -0.06 & $-7.11 *$ \\
\hline 9 & CAK 53A X AKH-07R & $1.75^{* *}$ & -2.71 & 0.04 & $-1.53 * *$ & 0.020 & $3.32 * *$ & $15.45 * *$ & $0.74 * *$ & $33.72 * *$ \\
\hline 10 & CAK 53A X DHY-286-1R & $-3.21 * *$ & -3.07 & 0.33 & $1.60 * *$ & $-0.035 * *$ & 0.28 & $-5.13 *$ & 0.17 & $-11.72 * *$ \\
\hline
\end{tabular}




\begin{tabular}{|c|c|c|c|c|c|c|c|c|c|c|}
\hline $\begin{array}{l}\text { Sr. } \\
\text { No. }\end{array}$ & Genotype & $\begin{array}{c}\text { Days to } \\
50 \% \\
\text { flowering }\end{array}$ & $\begin{array}{c}\text { Plant } \\
\text { height } \\
(\mathbf{c m})\end{array}$ & $\begin{array}{c}\text { No. of } \\
\text { monopodia } \\
\text { per plant }\end{array}$ & $\begin{array}{l}\text { Days to } \\
50 \% \text { boll } \\
\text { burst }\end{array}$ & $\begin{array}{l}\text { Earliness } \\
\text { index }\end{array}$ & $\begin{array}{c}\text { No. of } \\
\text { sympodia } \\
\text { per plant }\end{array}$ & $\begin{array}{l}\text { No. of bolls } \\
\text { per plant }\end{array}$ & $\begin{array}{c}\text { Boll } \\
\text { weight } \\
\text { (g) }\end{array}$ & $\begin{array}{c}\text { Seed cotton } \\
\text { yield } \\
\text { per plant } \\
\text { (g) }\end{array}$ \\
\hline 11 & CAK 53B X R-42-8 & 0.18 & $6.39 *$ & 0.12 & 0.48 & $-0.033^{*}$ & 0.77 & $5.09 *$ & 0.10 & $-11.61 * *$ \\
\hline 12 & CAK 53B X R-2000-23 & 0.57 & 1.43 & 0.11 & $1.62 * *$ & $0.048 * *$ & 0.59 & -3.70 & 0.06 & $12.03 * *$ \\
\hline 13 & CAK 53B X R- 2000-17-2 & $-2.70 * *$ & 2.34 & -0.13 & $-3.35^{* *}$ & $0.032 *$ & -0.13 & $4.59 *$ & 0.14 & 0.55 \\
\hline 14 & CAK 53B X R-2000-26 & $-1.64 * *$ & 1.06 & 0.24 & -1.10 & 0.020 & 1.41 & $6.12 * *$ & 0.03 & 6.10 \\
\hline 15 & CAK 53B X R-1044-13 & $6.80 * *$ & 1.30 & 0.12 & $4.62 * *$ & $-0.033^{*}$ & -0.14 & $-5.26^{*}$ & -0.28 & $-29.64 * *$ \\
\hline 16 & CAK 53B X R-2000-21 & $2.77 * *$ & -2.61 & 0.19 & -0.10 & $0.039 * *$ & 0.84 & 0.30 & -0.37 & $-10.01 * *$ \\
\hline 17 & CAK 53B X R-23 & $-1.50 * *$ & $-5.98 *$ & $-0.42 *$ & $-2.23 * *$ & $-0.034 * *$ & -0.04 & 0.50 & 0.09 & $23.62 * *$ \\
\hline 18 & CAK 53B X R-53 & $-1.72 * *$ & 0.23 & 0.008 & $-1.37 *$ & -0.010 & -1.39 & -0.59 & $0.61 * *$ & $11.00 * *$ \\
\hline 19 & CAK 53B X AKH-07R & 0.02 & 2.04 & -0.35 & $1.15^{*}$ & 0.008 & -1.35 & -0.13 & $-0.45^{*}$ & -5.24 \\
\hline 20 & CAK 53B X DHY-286-1R & $-2.78 * *$ & $-6.22 *$ & 0.10 & 0.28 & $-0.037 * *$ & -0.54 & $-6.91 * *$ & 0.05 & 3.19 \\
\hline 21 & CAK 23A X R-42-8 & $-6.74 * *$ & $-6.62 *$ & -0.09 & $-4.75^{* *}$ & $0.091 * *$ & 1.23 & 2.78 & 0.02 & $45.89 * *$ \\
\hline 22 & CAK 23A X R-2000-23 & $-4.18 * *$ & -1.45 & -0.06 & $-1.77 * *$ & $-0.026^{*}$ & 0.92 & 3.63 & -0.24 & $27.92 * *$ \\
\hline 23 & CAK 23A X R- 2000-17-2 & $2.86^{* *}$ & 0.24 & 0.26 & $2.25^{* *}$ & $-0.049 * *$ & 0.63 & 1.71 & 0.06 & $17.40 * *$ \\
\hline 24 & CAK 23A X R-2000-26 & $6.08 * *$ & $-6.03 *$ & -0.28 & $1.33 *$ & 0.004 & -0.11 & -3.79 & 0.14 & $-41.49 * *$ \\
\hline 25 & CAK 23A X R-1044-13 & $-3.96^{* *}$ & -1.81 & 0.35 & $-1.77 * *$ & -0.016 & 1.13 & 0.65 & 0.35 & $15.92 * *$ \\
\hline 26 & CAK 23A X R-2000-21 & -0.16 & -4.56 & $-0.44^{*}$ & $2.00 * *$ & -0.026 & 0.55 & 1.19 & 0.25 & $8.55^{* *}$ \\
\hline 27 & CAK 23A X R-23 & $1.39 *$ & 2.92 & -0.15 & 1.02 & 0.005 & -0.72 & -1.17 & 0.25 & $-15.80 * *$ \\
\hline 28 & CAK 23A X R-53 & $3.00 * *$ & 3.68 & 0.04 & 0.05 & 0.014 & 0.28 & -2.19 & -0.12 & -2.84 \\
\hline 29 & CAK 23A X AKH-07R & -0.24 & $7.10^{*}$ & $0.79 * *$ & 0.25 & $-0.066 * *$ & $-2.56^{* *}$ & $-5.47 * *$ & $-0.58 * *$ & $-36.51 * *$ \\
\hline 30 & CAK 23A X DHY-286-1R & $1.95 * *$ & $6.54 *$ & $-0.42 *$ & $1.38 *$ & $0.070 * *$ & -1.34 & 2.66 & -0.15 & $-19.04 * *$ \\
\hline 31 & CAK 23B X R-42-8 & $-2.16^{* *}$ & $-7.57 * *$ & 0.29 & $-1.31^{*}$ & $-0.036 * *$ & $-2.83 * *$ & $-5.44 * *$ & 0.17 & $-43.23 * *$ \\
\hline 32 & CAK 23B X R-2000-23 & 0.22 & $10.61 * *$ & -0.03 & $1.15^{*}$ & $-0.029 *$ & $2.15^{*}$ & $10.92 * *$ & 0.04 & $15.88 * *$ \\
\hline 33 & CAK 23B X R- 2000-17-2 & 0.78 & 4.49 & 0.22 & $1.51 * *$ & $-0.067 * *$ & -0.26 & $-7.32 * *$ & $-0.50^{*}$ & -3.22 \\
\hline 34 & CAK 23B X R-2000-26 & $-1.82 * *$ & 4.36 & -0.15 & -0.73 & -0.019 & $-2.09 *$ & $-5.80 * *$ & $-0.50^{*}$ & $-26.68 * *$ \\
\hline 35 & CAK 23B X R-1044-13 & 0.95 & $9.76^{* *}$ & -0.14 & $-1.67 * *$ & 0.004 & -0.89 & 0.69 & -0.03 & $18.86^{* *}$ \\
\hline 36 & CAK 23B X R-2000-21 & $-2.07 * *$ & 2.18 & -0.16 & $-2.23 * *$ & 0.010 & 0.41 & -1.68 & 0.008 & $-11.89 * *$ \\
\hline 37 & CAK 23B X R-23 & $1.14^{*}$ & $-10.97 * *$ & 0.02 & 0.96 & $0.030 *$ & -0.01 & -1.83 & 0.18 & -1.74 \\
\hline 38 & CAK 23B X R-53 & $1.25^{*}$ & $-9.06 * *$ & -0.16 & $1.32 *$ & 0.021 & -0.45 & $-5.00^{*}$ & 0.06 & -1.16 \\
\hline 39 & CAK 23B X AKH-07R & 0.33 & -5.42 & -0.12 & $-1.15^{*}$ & -0.001 & $1.92 *$ & $5.83 * *$ & $0.52 *$ & 5.22 \\
\hline 40 & CAK 23B X DHY-286-1R & $1.36^{*}$ & 1.62 & 0.24 & $2.15^{* *}$ & $0.086^{* *}$ & $2.06^{*}$ & $9.65 * *$ & 0.04 & $47.99 * *$ \\
\hline
\end{tabular}


Table 1 Continue...

\begin{tabular}{|c|c|c|c|c|c|c|c|c|c|c|}
\hline 41 & SRT-1A X R-42-8 & $4.23 * *$ & $-12.52 * *$ & -0.01 & $3.40 * *$ & -0.019 & -0.84 & $-4.87 *$ & -0.20 & $18.69 * *$ \\
\hline $\begin{array}{l}\text { Sr. } \\
\text { No. }\end{array}$ & Genotype & $\begin{array}{c}\text { Days to } \\
50 \% \\
\text { flowering }\end{array}$ & $\begin{array}{c}\text { Plant } \\
\text { height } \\
\text { (cm) }\end{array}$ & $\begin{array}{c}\text { No. of } \\
\text { monopodia } \\
\text { per plant }\end{array}$ & $\begin{array}{c}\text { Days to } \\
50 \% \text { boll } \\
\text { burst }\end{array}$ & $\begin{array}{c}\text { Earliness } \\
\text { index }\end{array}$ & $\begin{array}{c}\text { No. of } \\
\text { sympodia } \\
\text { per plant }\end{array}$ & $\begin{array}{c}\text { No. of bolls } \\
\text { per plant }\end{array}$ & $\begin{array}{c}\text { Boll } \\
\text { weight } \\
\text { (g) }\end{array}$ & $\begin{array}{l}\text { Seed cotton } \\
\text { yield } \\
\text { per plant } \\
\text { (g) }\end{array}$ \\
\hline 42 & SRT-1A X R-2000-23 & $3.96 * *$ & $-13.22 * *$ & -0.06 & $2.87 * *$ & 0.012 & -1.05 & $-11.61 * *$ & 0.19 & $-27.36 * *$ \\
\hline 43 & SRT-1A X R- 2000-17-2 & $3.68 * *$ & $-6.51 *$ & 0.02 & 0.90 & 0.020 & 0.46 & -0.25 & 0.06 & $-15.39 * *$ \\
\hline 44 & SRT-1A X R-2000-26 & 0.90 & -1.35 & 0.17 & $1.31 *$ & -0.021 & $2.80 * *$ & $9.14 * *$ & $0.54 * *$ & $42.89 * *$ \\
\hline 45 & SRT-1A X R-1044-13 & $-5.65 * *$ & -4.57 & -0.01 & $-3.79 * *$ & $0.069 * *$ & 0.31 & -0.83 & 0.12 & 5.01 \\
\hline 46 & SRT-1A X R-2000-21 & $-4.17 * *$ & 3.47 & 0.36 & $-3.51 * *$ & -0.002 & 0.35 & 2.48 & 0.02 & $14.99 * *$ \\
\hline 47 & SRT-1A X R-23 & $-4.78 * *$ & $10.30 * *$ & -0.08 & -0.15 & -0.005 & 0.67 & 2.44 & $-0.47 *$ & $-23.41 * *$ \\
\hline 48 & SRT-1A X R-53 & -1.01 & $8.84 * *$ & -0.29 & 0.70 & -0.019 & 0.27 & $5.33 *$ & -0.29 & $-18.69 * *$ \\
\hline 49 & SRT-1A X AKH-07R & -0.42 & $9.70 * *$ & -0.21 & 0.90 & $0.026 *$ & -1.68 & -3.32 & -0.07 & $10.64 *$ \\
\hline 50 & SRT-1A X DHY-286-1R & $3.26 * *$ & $5.86 *$ & 0.12 & $-2.62 * *$ & $-0.061 * *$ & -1.31 & 1.50 & 0.08 & $-7.36 *$ \\
\hline 51 & SRT-1B X R-42-8 & $-2.61 * *$ & $16.75 * *$ & -0.13 & $-1.28 *$ & -0.018 & -0.14 & 1.60 & 0.08 & $6.51 *$ \\
\hline 52 & SRT-1B X R-2000-23 & $-2.22 * *$ & $8.05 * *$ & 0.19 & $-2.81 * *$ & $-0.027 *$ & -0.40 & $4.20 *$ & 0.07 & 0.009 \\
\hline 53 & SRT-1B X R- 2000-17-2 & -0.66 & -2.41 & 0.04 & 0.55 & $0.066 * *$ & 0.15 & 1.55 & 0.25 & $7.47 *$ \\
\hline 54 & SRT-1B X R-2000-26 & $2.88 * *$ & 4.80 & 0.30 & $1.96 * *$ & -0.007 & $-1.98^{*}$ & -2.87 & -0.13 & -3.17 \\
\hline 55 & SRT-1B X R-1044-13 & 0.33 & $-7.12 *$ & -0.10 & $2.02 * *$ & 0.008 & -0.30 & $6.437 * *$ & 0.20 & $-24.19 * *$ \\
\hline 56 & SRT-1B X R-2000-21 & 0.97 & 1.60 & -0.05 & $2.46^{* *}$ & $-0.041 * *$ & -1.78 & -3.79 & 0.00 & 3.04 \\
\hline 57 & SRT-1B X R-23 & $1.86^{* *}$ & 0.26 & 0.02 & 0.66 & $0.032 *$ & 0.90 & 2.48 & 0.07 & $12.41 * *$ \\
\hline 58 & SRT-1B X R-53 & $1.47 * *$ & $-6.51 *$ & 0.25 & $-1.14 *$ & -0.003 & $2.38^{*}$ & $4.51 *$ & -0.19 & $18.81 * *$ \\
\hline 59 & SRT-1B X AKH-07R & $-1.44 * *$ & $-10.70 * *$ & -0.13 & 0.38 & 0.012 & 0.36 & $-12.36 * *$ & -0.15 & $-7.83^{*}$ \\
\hline \multirow[t]{5}{*}{60} & SRT-1B X DHY-286-1R & -0.58 & -4.73 & $-0.38 *$ & $-2.81 * *$ & -0.022 & 0.85 & -1.76 & -0.20 & $-13.05 * *$ \\
\hline & S.E. \pm & & & & & & & & & \\
\hline & Lines & 0.17 & 0.88 & 0.06 & 0.18 & 0.004 & 0.31 & 0.65 & 0.06 & 1.00 \\
\hline & Tester & 0.22 & 1.14 & 0.08 & 0.23 & 0.005 & 0.39 & 0.84 & 0.08 & 1.29 \\
\hline & Crosses & 0.55 & 2.78 & 0.18 & 0.56 & 0.013 & 0.97 & 2.06 & 0.20 & 3.17 \\
\hline
\end{tabular}


Table 1 Continue...

\begin{tabular}{|c|c|c|c|c|c|c|c|c|c|c|}
\hline $\begin{array}{l}\text { Sr. } \\
\text { No. }\end{array}$ & Genotype & $\begin{array}{c}\text { Seed } \\
\text { index }(g)\end{array}$ & $\begin{array}{c}\text { Lint } \\
\text { index }(g)\end{array}$ & $\begin{array}{c}\text { Harvest } \\
\text { index }(\%)\end{array}$ & $\begin{array}{l}\text { Seed cotton } \\
\text { yield per } \\
\text { hectare }(\mathrm{kg})\end{array}$ & $\begin{array}{c}\text { Ginning } \\
\text { percentage }\end{array}$ & $\begin{array}{c}\text { Upper half } \\
\text { mean } \\
\text { length } \\
(\mathrm{mm})\end{array}$ & $\begin{array}{l}\text { Uniformity } \\
\text { index }(\%)\end{array}$ & $\begin{array}{c}\text { Micronaire } \\
\text { value (ug/in) }\end{array}$ & $\begin{array}{c}\text { Fibre } \\
\text { strength } \\
\text { (g/tex) }\end{array}$ \\
\hline & GCA Lines & & & & & & & & & \\
\hline 1 & CAK 53A & $-0.33 * *$ & -0.07 & $-1.55 * *$ & $-148.06 * *$ & -0.07 & $0.40 * *$ & -0.06 & $-0.30 * *$ & $-0.31 * *$ \\
\hline 2 & CAK 53B & 0.01 & -0.09 & $-0.46^{*}$ & -4.45 & -0.12 & $0.89 * *$ & $0.69 * *$ & $-0.14 * *$ & $0.63 * *$ \\
\hline 3 & CAK 23A & 0.12 & $-0.16^{*}$ & $-1.00 * *$ & $-160.81 * *$ & $-0.57 * *$ & $-0.47 * *$ & $-0.64 * *$ & $0.18 * *$ & 0.03 \\
\hline 4 & CAK 23B & 0.008 & $0.16^{*}$ & $4.52 * *$ & $231.32 * *$ & $0.45 * *$ & $0.52 * *$ & $0.39 * *$ & $-0.18 * *$ & $1.12 * *$ \\
\hline 5 & SRT-1A & 0.04 & 0.03 & $-1.16 * *$ & $-85.16 * *$ & -0.07 & $-1.08 * *$ & -0.15 & $0.15^{* *}$ & $-1.26 * *$ \\
\hline \multirow[t]{2}{*}{6} & SRT-1B & 0.14 & 0.12 & -0.33 & $167.18 * *$ & $0.38 *$ & $-0.27 * *$ & $-0.22 *$ & $0.27 * *$ & $-0.21 *$ \\
\hline & GCA Testers & & & & & & & & & \\
\hline 1 & $\mathrm{R}-42-8$ & -0.15 & -0.04 & -0.52 & -37.20 & $0.62 * *$ & $-0.47 * *$ & $-0.96 * *$ & $0.40 * *$ & $-1.13 * *$ \\
\hline 2 & R-2000-23 & -0.02 & 0.02 & $1.75 * *$ & $269.78 * *$ & $0.81 * *$ & $1.12 * *$ & $1.16 * *$ & $-0.16 * *$ & $1.68 * *$ \\
\hline 3 & R- 2000-17-2 & 0.04 & -0.14 & $-0.81 * *$ & $-172.19 * *$ & $-0.75 * *$ & $0.84 * *$ & $0.67 * *$ & $-0.26 * *$ & -0.09 \\
\hline 4 & R-2000-26 & 0.18 & $0.37 * *$ & $1.87 * *$ & $74.16 * *$ & $0.81 * *$ & $0.17 *$ & 0.23 & $-0.19 * *$ & $-1.00 * *$ \\
\hline 5 & R-1044-13 & 0.01 & $-0.22 *$ & $-0.75 * *$ & 32.83 & $-1.03 * *$ & $0.80 * *$ & 0.08 & $-0.28 * *$ & $0.75 * *$ \\
\hline 6 & $\mathrm{R}-2000-21$ & $-0.20 *$ & -0.07 & $-0.69 *$ & $-200.314 * *$ & $0.66 * *$ & $-0.97 * *$ & $-1.18 * *$ & $-0.09 *$ & $-0.40 * *$ \\
\hline 7 & $\mathrm{R}-23$ & -0.03 & -0.16 & $-3.49 * *$ & $-269.09 * *$ & $-0.67 * *$ & $-1.03 * *$ & $0.37 * *$ & $0.20 * *$ & $-0.71 * *$ \\
\hline 8 & $\mathrm{R}-53$ & -0.18 & -0.13 & 0.08 & 17.34 & $-0.48 *$ & $-0.73 * *$ & $0.30 *$ & -0.05 & $0.43 * *$ \\
\hline 9 & AKH-07R & 0.11 & $0.21 *$ & $3.79 * *$ & $547.42 * *$ & $-0.55 * *$ & $-0.86 * *$ & $-0.60 * *$ & 0.05 & $-0.52 * *$ \\
\hline \multirow[t]{2}{*}{10} & DHY-286-1R & $0.24 * *$ & 0.16 & $-1.21 * *$ & $-262.74 * *$ & $0.58 * *$ & $1.13 * *$ & -0.08 & $0.40 * *$ & $1.00 * *$ \\
\hline & SCA Crosses & & & & & & & & & \\
\hline 1 & CAK 53A X R-42-8 & $-0.77 * *$ & $-0.48 *$ & $1.57 *$ & $-300.93 * *$ & 0.50 & $-2.07 * *$ & $-1.82 * *$ & 0.12 & $-1.09 * *$ \\
\hline 2 & CAK 53A X R-2000-23 & $0.54 *$ & -0.02 & $-6.58 * *$ & $-527.64 * *$ & 0.19 & 0.04 & $-0.63 *$ & 0.12 & -0.45 \\
\hline 3 & CAK 53A X R- 2000-17-2 & $-0.62 * *$ & -0.30 & $2.12 * *$ & $-126.00 *$ & $-1.89 * *$ & 0.16 & 0.22 & $0.30 * *$ & $-0.68 *$ \\
\hline 4 & CAK 53A X R-2000-26 & $0.47^{*}$ & 0.14 & 0.25 & $414.12 * *$ & -0.37 & $-0.69 * *$ & $-1.16 * *$ & $-0.43 * *$ & 0.10 \\
\hline 5 & CAK 53A X R-1044-13 & 0.26 & -0.003 & $-6.97 * *$ & $260.05 * *$ & -0.80 & $-0.78 * *$ & $0.82 * *$ & 0.09 & $-0.92 * *$ \\
\hline 6 & CAK 53A X R-2000-21 & -0.05 & -0.03 & $4.03 * *$ & -86.56 & 0.06 & $0.44 *$ & $1.16^{* *}$ & -0.18 & $0.56^{*}$ \\
\hline 7 & CAK 53A X R-23 & $0.50 *$ & -0.18 & $-1.67 *$ & 91.22 & 0.39 & $3.02 * *$ & $0.63 *$ & $0.47 * *$ & $1.01 * *$ \\
\hline 8 & CAK 53A X R-53 & 0.28 & 0.066 & $2.55^{* *}$ & $-131.69 *$ & $-1.14^{*}$ & $0.77 * *$ & 0.53 & 0.02 & $1.18 * *$ \\
\hline
\end{tabular}


Table 1 Continue...

\begin{tabular}{|c|c|c|c|c|c|c|c|c|c|c|}
\hline 9 & CAK 53A X AKH-07R & 0.005 & $0.96 * *$ & $9.66 * *$ & $624.54 * *$ & $2.72 * *$ & $1.00 * *$ & $1.51 * *$ & 0.02 & 0.05 \\
\hline 10 & CAK 53A X DHY-286-1R & $-0.61 * *$ & -0.13 & $-4.97 * *$ & $-217.09 * *$ & 0.31 & $-1.91 * *$ & $-1.27 * *$ & $-0.54 * *$ & 0.23 \\
\hline 11 & CAK 53B X R-42-8 & $-0.78 * *$ & -0.12 & $-1.32 *$ & $-214.99 * *$ & $1.19 *$ & $0.92 * *$ & $1.17 * *$ & $0.19 *$ & 0.36 \\
\hline 12 & CAK 53B X R-2000-23 & $-0.99 * *$ & $-0.45^{*}$ & -0.28 & $222.87 * *$ & $-1.66 * *$ & 0.26 & -0.07 & -0.16 & $3.39 * *$ \\
\hline $\begin{array}{l}\text { Sr. } \\
\text { No. }\end{array}$ & Genotype & $\begin{array}{c}\text { Seed } \\
\text { index }(\mathrm{g})\end{array}$ & $\begin{array}{c}\text { Lint } \\
\text { index (g) }\end{array}$ & $\begin{array}{c}\text { Harvest } \\
\text { index }(\%)\end{array}$ & $\begin{array}{l}\text { Seed cotton } \\
\text { yield per } \\
\text { hectare } \\
(\mathbf{k g})\end{array}$ & $\begin{array}{c}\text { Ginning } \\
\text { percentage }\end{array}$ & $\begin{array}{c}\text { Upper } \\
\text { half mean } \\
\text { length } \\
\text { (mm) }\end{array}$ & $\begin{array}{l}\text { Uniformity } \\
\text { index }(\%)\end{array}$ & $\begin{array}{c}\text { Micronaire } \\
\text { value } \\
\text { (ug/in) }\end{array}$ & $\begin{array}{c}\text { Fibre } \\
\text { strength } \\
\text { (g/tex) }\end{array}$ \\
\hline 13 & CAK 53B X R- 2000-17-2 & $0.48 *$ & 0.23 & $3.47 * *$ & 10.24 & -0.22 & -0.33 & $-1.13 * *$ & 0.11 & 0.50 \\
\hline 14 & CAK 53B X R-2000-26 & -0.11 & 0.03 & $-2.73 * *$ & 113.00 & 0.05 & $-1.46 * *$ & $-1.06 * *$ & -0.01 & $-2.36 * *$ \\
\hline 15 & CAK 53B X R-1044-13 & 0.16 & -0.15 & $-3.03 * *$ & $-548.94 * *$ & $-1.93 * *$ & -0.16 & $2.14 * *$ & -0.01 & 0.29 \\
\hline 16 & CAK 53B X R-2000-21 & $0.87 * *$ & $0.46^{*}$ & $-4.02 * *$ & $-185.52 * *$ & 0.55 & 0.07 & $0.64 *$ & 0.10 & $-0.84 * *$ \\
\hline 17 & CAK 53B X R-23 & $-0.69 * *$ & -0.20 & 0.35 & $437.53 * *$ & $1.33 * *$ & $0.54 * *$ & 0.57 & -0.14 & -0.18 \\
\hline 18 & CAK 53B X R-53 & $0.56^{*}$ & $0.45^{*}$ & $6.90 * *$ & $203.79 * *$ & $1.08 *$ & $0.46 * *$ & $-0.63^{*}$ & $-0.18 *$ & $-1.08 * *$ \\
\hline 19 & CAK 53B X AKH-07R & 0.23 & -0.33 & -0.80 & -97.11 & 0.92 & 0.20 & 0.01 & -0.04 & -0.07 \\
\hline 20 & CAK 53B X DHY-286-1R & 0.26 & 0.09 & $1.47 *$ & 59.12 & $-1.31 * *$ & $-0.49 * *$ & $-1.64 * *$ & 0.16 & -0.008 \\
\hline 21 & CAK 23A X R-42-8 & $0.58 *$ & 0.24 & 0.93 & $849.83 * *$ & -0.40 & $-1.64 * *$ & $1.32 * *$ & -0.08 & $-2.96 * *$ \\
\hline 22 & CAK 23A X R-2000-23 & -0.24 & -0.05 & $-3.51 * *$ & $517.17 * *$ & 0.83 & -0.10 & 0.52 & $-0.27 * *$ & $-2.00 * *$ \\
\hline 23 & CAK 23A X R- 2000-17-2 & -0.03 & 0.19 & $1.62 *$ & $322.22 * *$ & $-1.12 *$ & $1.04 * *$ & $1.72 * *$ & $-0.26 * *$ & $1.85 * *$ \\
\hline 24 & CAK 23A X R-2000-26 & 0.06 & 0.22 & $1.41 *$ & $-768.46 * *$ & $1.42 * *$ & $-0.58 * *$ & $-0.65^{*}$ & $0.28 * *$ & 0.42 \\
\hline 25 & CAK 23A X R-1044-13 & 0.12 & 0.03 & $2.06 * *$ & $294.87 * *$ & -0.49 & -0.318 & -0.52 & $0.59 * *$ & $-2.88 * *$ \\
\hline 26 & CAK 23A X R-2000-21 & -0.39 & -0.13 & 0.98 & $158.46^{* *}$ & 0.11 & $2.41 * *$ & -0.03 & $-0.20^{*}$ & $2.26 * *$ \\
\hline 27 & CAK 23A X R-23 & -0.28 & -0.03 & 1.15 & $-292.65 * *$ & -0.07 & $-1.37 * *$ & 0.31 & $-0.34 * *$ & $1.06 * *$ \\
\hline 28 & CAK 23A X R-53 & 0.19 & -0.41 & $-1.97 * *$ & -52.68 & $-1.22 *$ & 0.20 & $-0.77 *$ & -0.11 & $2.10 * *$ \\
\hline 29 & CAK 23A X AKH-07R & -0.01 & -0.16 & $-6.12 * *$ & $-676.15 * *$ & -0.41 & $-1.04 * *$ & $-1.93 * *$ & $0.28 * *$ & $-0.69 *$ \\
\hline 30 & CAK 23A X DHY-286-1R & 0.01 & 0.11 & $3.43 * *$ & $-352.61 * *$ & $1.35 * *$ & $1.41 * *$ & 0.03 & 0.12 & $0.83 * *$ \\
\hline 31 & CAK 23B X R-42-8 & $0.49 *$ & 0.21 & $-1.83 * *$ & $-800.69 * *$ & -0.29 & $-0.78 * *$ & $-1.42 * *$ & $-0.44 * *$ & $0.97 * *$ \\
\hline 32 & CAK 23B X R-2000-23 & $0.46^{*}$ & 0.36 & $2.39 * *$ & $294.16^{* *}$ & 0.46 & $-0.91 * *$ & $-1.42 * *$ & 0.04 & $-3.10 * *$ \\
\hline 33 & CAK 23B X R- 2000-17-2 & 0.14 & -0.37 & $-3.04 * *$ & -59.76 & $-1.25^{*}$ & $1.31 * *$ & $0.88 * *$ & $0.18^{*}$ & $-0.80 * *$ \\
\hline 34 & CAK 23B X R-2000-26 & $-0.45^{*}$ & $-0.60 * *$ & $-3.82 * *$ & $-494.14 * *$ & $-1.40 * *$ & $1.41 * *$ & $1.16^{* *}$ & $0.31 * *$ & $0.98 * *$ \\
\hline 35 & CAK 23B X R-1044-13 & $0.49 *$ & 0.14 & $-1.53 *$ & $349.26 * *$ & 0.76 & $0.94 * *$ & $-0.61 *$ & $-0.55 * *$ & $2.16 * *$ \\
\hline 36 & CAK 23B X R-2000-21 & $-0.53 *$ & -0.37 & 0.67 & $-220.34 * *$ & 0.36 & $-1.34 * *$ & $-0.61 *$ & $0.40 * *$ & -0.21 \\
\hline 37 & CAK 23B X R-23 & 0.02 & 0.05 & $1.45^{*}$ & -32.37 & $0.96^{*}$ & $-0.92 * *$ & -0.56 & -0.06 & 0.10 \\
\hline 38 & CAK 23B X R-53 & $-0.56^{*}$ & -0.40 & $-1.73 * *$ & -21.62 & $1.23^{*}$ & -0.30 & 0.05 & -0.15 & $0.87 * *$ \\
\hline
\end{tabular}


Table 1 Continue...

\begin{tabular}{|c|c|c|c|c|c|c|c|c|c|c|}
\hline 39 & CAK 23B X AKH-07R & $-0.68 * *$ & $0.47 *$ & $1.44^{*}$ & 96.82 & $-1.07 *$ & $0.98 * *$ & $1.86 * *$ & -0.07 & $1.38 * *$ \\
\hline 40 & CAK 23B X DHY-286-1R & $0.60 * *$ & $0.49 *$ & $5.99 * *$ & $888.69 * *$ & 0.22 & $-0.39 *$ & $0.67 *$ & $0.33 * *$ & $-2.36 * *$ \\
\hline 41 & SRT-1A X R-42-8 & 0.03 & 0.01 & -0.05 & $346.17 * *$ & 0.47 & $1.05 * *$ & -0.34 & 0.07 & $1.31 * *$ \\
\hline 42 & SRT-1A X R-2000-23 & -0.03 & -0.04 & $2.39 * *$ & $-506.72 * *$ & -0.32 & $2.45 * *$ & $1.65^{*}$ & -0.04 & $3.59 * *$ \\
\hline 43 & SRT-1A X R-2000-17-2 & 0.10 & 0.22 & $-1.84 * *$ & $-285.11 * *$ & $2.35 * *$ & -0.19 & -0.50 & 0.07 & $-0.95 * *$ \\
\hline $\begin{array}{l}\text { Sr. } \\
\text { No. }\end{array}$ & Genotype & $\begin{array}{c}\text { Seed } \\
\text { index }(g)\end{array}$ & $\begin{array}{c}\text { Lint } \\
\text { index }(g)\end{array}$ & $\begin{array}{l}\text { Harvest } \\
\text { index (g) }\end{array}$ & $\begin{array}{l}\text { Seed cotton } \\
\text { yield per } \\
\text { hectare } \\
(\mathbf{k g})\end{array}$ & $\begin{array}{c}\text { Ginning } \\
\text { percentage }\end{array}$ & $\begin{array}{c}\text { Upper } \\
\text { half mean } \\
\text { length } \\
(\mathrm{mm})\end{array}$ & $\begin{array}{l}\text { Uniformity } \\
\text { index }(\%)\end{array}$ & $\begin{array}{c}\text { Micronaire } \\
\text { value } \\
\text { (ug/in) }\end{array}$ & $\begin{array}{c}\text { Fibre } \\
\text { strength } \\
\text { (g/tex) }\end{array}$ \\
\hline 44 & SRT-1A X R-2000-26 & 0.02 & 0.38 & $7.88 * *$ & $794.35 * *$ & $1.02 *$ & $-0.54 * *$ & $0.86 * *$ & -0.07 & $-0.66^{*}$ \\
\hline 45 & SRT-1A X R-1044-13 & $-0.71 * *$ & -0.17 & $5.37 * *$ & 92.80 & 0.95 & $-0.59 * *$ & $-1.87 * *$ & 0.09 & $-1.03 * *$ \\
\hline 46 & SRT-1A X R-2000-21 & 0.07 & 0.009 & -0.33 & $277.58 * *$ & $-1.39 * *$ & $0.66 * *$ & 0.27 & $-0.29 * *$ & -0.47 \\
\hline 47 & SRT-1A X R-23 & 0.17 & 0.14 & $-3.66 * *$ & $-433.58 * *$ & $-1.49 * *$ & $-0.93 * *$ & 0.21 & 0.06 & $-0.82 * *$ \\
\hline 48 & SRT-1A X R-53 & -0.03 & 0.19 & $-4.70 * *$ & $-346.16 * *$ & $-1.53 * *$ & $-0.89 * *$ & $0.78 *$ & -0.03 & $-1.61 * *$ \\
\hline 49 & SRT-1A X AKH-07R & 0.33 & $-0.49 *$ & -0.97 & $197.05 * *$ & -0.44 & $-1.65 * *$ & $-2.17 * *$ & 0.12 & -0.38 \\
\hline 50 & SRT-1A X DHY-286-1R & 0.03 & -0.24 & $-4.07 * *$ & $-136.37 *$ & 0.37 & $0.65 * *$ & $1.11 * *$ & 0.01 & $1.03 * *$ \\
\hline 51 & SRT-1B X R-42-8 & 0.44 & 0.13 & 0.70 & $120.61 *$ & $-1.47 * *$ & $2.52 * *$ & $1.09 * *$ & 0.13 & $1.40 * *$ \\
\hline 52 & SRT-1B X R-2000-23 & 0.26 & 0.22 & $5.59 * *$ & 0.16 & 0.49 & $-1.73 * *$ & -0.05 & $0.31 * *$ & $-1.43 * *$ \\
\hline 53 & SRT-1B X R- 2000-17-2 & -0.08 & 0.01 & $-2.34 * *$ & $138.41 *$ & $2.13 * *$ & $-1.99 * *$ & $-1.18 * *$ & $-0.40 * *$ & 0.08 \\
\hline 54 & SRT-1B X R-2000-26 & 0.01 & -0.16 & $-2.99 * *$ & -58.87 & -0.72 & $1.87 * *$ & $0.85 * *$ & -0.07 & $1.50 * *$ \\
\hline 55 & SRT-1B X R-1044-13 & -0.34 & 0.15 & $4.10 * *$ & $-448.04 * *$ & $1.51 * *$ & $0.92 * *$ & 0.04 & $-0.21 *$ & $2.37 * *$ \\
\hline 56 & SRT-1B X R-2000-21 & 0.04 & 0.08 & $-1.34 *$ & 56.38 & 0.29 & $-2.25 * *$ & $-1.42 * *$ & 0.16 & $-1.29 * *$ \\
\hline 57 & SRT-1B X R-23 & 0.27 & 0.24 & $2.38 * *$ & $229.84 * *$ & $-1.12 *$ & -0.32 & $-1.18 * *$ & 0.01 & $-1.18 * *$ \\
\hline 58 & SRT-1B X R-53 & -0.44 & 0.10 & -1.05 & $348.37 * *$ & $1.58 * *$ & -0.24 & 0.03 & $0.46^{* *}$ & $-1.46^{* *}$ \\
\hline 59 & SRT-1B X AKH-07R & 0.12 & $-0.45^{*}$ & $-3.20 * *$ & $-145.14^{*}$ & $-1.71 * *$ & $0.51 * *$ & $0.72 *$ & $-0.32 * *$ & -0.27 \\
\hline \multirow[t]{5}{*}{60} & SRT-1B X DHY-286-1R & -0.30 & -0.31 & $-1.84 * *$ & $-241.73 * *$ & $-0.96 *$ & $0.72 * *$ & $1.09 * *$ & -0.08 & 0.26 \\
\hline & S.E. \pm & & & & & & & & & \\
\hline & Lines & 0.07 & 0.07 & 0.21 & 18.56 & 0.15 & 0.06 & 0.10 & 0.03 & 0.09 \\
\hline & Tester & 0.09 & 0.09 & 0.27 & 23.96 & 0.20 & 0.07 & 0.13 & 0.04 & 0.11 \\
\hline & Crosses & 0.23 & 0.22 & 0.66 & 58.68 & 0.49 & 0.18 & 0.31 & 0.09 & 0.28 \\
\hline
\end{tabular}


Selection of the most encouraging cross combination needs high SCA along with high per se and GCA effect of respective parents. The cross combinations CAK 53A x AKH07R, CAK 23B x AKH-07R, CAK 23B x DHY-286-1R, CAK 23B x R-2000-23, SRT1A x R-2000-26, CAK 23A x R-42-8, CAK 53B x R-23 and SRT-1A x R-2000-26 shows high SCA effect for seed cotton yield. For fibre strength CAK 53 B x R-2000-23, SRT1A x R-2000-23 possessed the high SCA effect. Ahuja and Dhayal (2007), Panhwaret al.(2008), Abroet al.(2009), Anandan (2010), Patilet al.(2011), Simon (2013), Patel and Kumar (2014), Pushpam et al.(2015) and Reddy et al.(2016).

\section{References}

Ahuja, S. L. and Dhayal, L. S. 2007. Combining ability estimates for yield and fibre quality traits in $4 \times 13$ line $\mathrm{x}$ tester crosses of $G$. hirsutum. Euphytica153: 87-98.

Panhwar, S. A., Baloch, M. J., Jatoi, W., Veesar, N. F. and Majeedano. 2008. Combining ability estimates from line $\mathrm{x}$ tester mating design in upland cotton. Proc. Pakistan Acad. Sci. 45 (2): 69-74.

Abro, S., Khandro, M. M., Laghari, M. A., Arain, M. A. and Deho, Z. A. 2009.Combining ability and hetrosis for yield contributing traits in upland cotton (G. hirsutum). Pak J. Bot.41(4):
1769-1774.

Anandan A. 2010. Environmental impact on the combining ability of fibre trait and seed - cotton yield in cotton. Journal of Crop Improvement 2: 310-323.

Patil, S.A., Naik, M. R., Chougule, G. R., Pathak, V. D. and Patil, A. B. 2011. Combining ability analysis for yield and fibre quality traits in upland cotton (Gossypium hirsutum L.). J. Cotton Res. Dev. 25 (2): 171-175.

Simon, S.Y., Kadams, A.M. and Aliyu, B. 2013.Combining ability analysis in $F_{1}$ hybrids of cotton by diallel method in Northeastern Nigeria. Greener J. of Agri. Sci. 3 (2): 90-96.

Patel, D. H., Patel, D. U. and Kumar, V. 2014.Hetrosis and combining ability analysis in tetraploid cotton (G.hirsutum and G.barbadense). Electronic J. of Plant Breeding 5 (3): 408-414.

Pushpam, R., Thangaraj, K. and Raveerandran, T. S. 2015. Heterosis and combining ability studies in upland cotton for yield characters. Electronic Journal of Plant Breeding. 6 (2): 459-463.

Reddy, K. B., Reddy, V. C., Ahmed, M. L., Naidu, T. C. M. and Srinivasarao, V. 2016.Combining ability study for yield and its component traits through diallel mating design in upland cotton (Gossypium hirsutum). J. Cotton Res. Dev. 30 (2) 180-184.

\section{How to cite this article:}

Gopal, G.R., D.B. Deosarkar and Chinchane, V.N. 2018. Combining Ability Studies in CMS based and Conventional Hybrids of Cotton (G. hirsutum). Int.J.Curr.Microbiol.App.Sci. 7(08): 4256-4265. doi: https://doi.org/10.20546/ijcmas.2018.708.446 